TRANSACTIONS OF THE

AMERICAN MATHEMATICAL SOCIETY

Volume 360, Number 2, February 2008, Pages 989-1004

S 0002-9947(07)04284-5

Article electronically published on May 11, 2007

\title{
GROMOV-WITTEN INVARIANTS OF JUMPING CURVES
}

\author{
IZZET COSKUN
}

A la memoire de Grandmaman Regine

\begin{abstract}
Given a vector bundle $E$ on a smooth projective variety $X$, we can define subschemes of the Kontsevich moduli space of genus-zero stable maps $M_{0,0}(X, \beta)$ parameterizing maps $f: \mathbb{P}^{1} \rightarrow X$ such that the Grothendieck decomposition of $f^{*} E$ has a specified splitting type. In this paper, using a "compactification" of this locus, we define Gromov-Witten invariants of jumping curves associated to the bundle $E$. We compute these invariants for the tautological bundle of Grassmannians and the Horrocks-Mumford bundle on $\mathbb{P}^{4}$. Our construction is a generalization of jumping lines for vector bundles on $\mathbb{P}^{n}$. Since for the tautological bundle of the Grassmannians the invariants are enumerative, we resolve the classical problem of computing the characteristic numbers of unbalanced scrolls.
\end{abstract}

\section{INTRODUCTION}

Given a vector bundle $E$ of rank $r$ on a smooth projective variety $X$ we define analogues of Gromov-Witten invariants for genus-zero jumping curves. We compute these invariants when $X$ is a Grassmannian and $E$ is its tautological bundle. Since these invariants are enumerative, we answer some classical questions about the characteristic numbers of scrolls. We also compute the invariants for the HorrocksMumford bundle.

A theorem of Grothendieck asserts that every vector bundle $E$ of rank $k$ on $\mathbb{P}^{1}$ decomposes as a direct sum of line bundles $E \cong \bigoplus_{i=1}^{k} \mathcal{O}_{\mathbb{P}^{1}}\left(r_{i}\right)$. If we assume that the $k$ integers $r_{1}, \ldots, r_{k}$ are in increasing order, then the isomorphism class of $E$ uniquely determines these integers ([GH] p. 516). We then say that $E$ has decomposition type $\left(r_{1}, \ldots, r_{k}\right)$.

Grothendieck's theorem provides a tool for understanding vector bundles on rationally connected varieties, especially on $\mathbb{P}^{n}$ (see [OSS]). If $E$ is a vector bundle on $\mathbb{P}^{n}$, by Grothendieck's theorem the restriction of $E$ to every line decomposes as a direct sum of line bundles. The semicontinuity of the dimension of cohomology groups implies that the decomposition type is constant on an open subset of the Grassmannian of lines $G(2, n+1)=\mathbb{G}(1, n)$. However, the decomposition type may change along subvarieties of $G(2, n+1)$. Lines that have decomposition types that differ from the general decomposition type are called jumping lines.

The subvarieties of jumping lines in $G(2, n+1)$ exhibit very interesting geometry and carry essential information about the vector bundle $E$. For example, if $E$ is the Horrocks-Mumford bundle on $\mathbb{P}^{4}[\mathrm{HM}]$, then the restriction of $E$ to a general line

Received by the editors May 14, 2005 and, in revised form, February 1, 2006.

2000 Mathematics Subject Classification. Primary 14F05, 14J60, 14N10, 14N35.

(C) 2007 American Mathematical Society Reverts to public domain 28 years from publication 
decomposes as $\mathcal{O}_{\mathbb{P}^{1}}(2) \oplus \mathcal{O}_{\mathbb{P}^{1}}(3)$. However, for lines contained in a rational four-fold in $G(2,5)$ the splitting is $\mathcal{O}_{\mathbb{P}^{1}}(1) \oplus \mathcal{O}_{\mathbb{P}^{1}}(4)$. The singular locus of this four-fold is a contraction of the Shioda modular surface $S(5)$ with 25 singular points. The lines of splitting type $\mathcal{O}_{\mathbb{P}^{1}} \oplus \mathcal{O}_{\mathbb{P}^{1}}(5)$ are parameterized by the surface, and the 25 singular points correspond to the lines with splitting $\mathcal{O}_{\mathbb{P}^{1}}(-1) \oplus \mathcal{O}_{\mathbb{P}^{1}}(6)$ (see $[\mathrm{Hu}$, [BHM]). Given the richness that jumping lines exhibit, it is natural to study the loci of higher degree rational curves where the decomposition of the bundle changes.

Jumping curves. Let $X$ be a smooth, projective variety. Let $E$ be a rank $k$ vector bundle on $X$. Let $\beta$ be a curve class on $X$. For any map $f: \mathbb{P}^{1} \rightarrow X$ in the Kontsevich space of $m$-pointed genus-zero maps $M_{0, m}(X, \beta)$ we say that $f$ has splitting type $\left(r_{1}, \ldots, r_{k}\right)$ for the vector bundle $E$ if the Grothendieck decomposition of $f^{*} E$ on $\mathbb{P}^{1}$ has type $\left(r_{1}, \ldots, r_{k}\right)$. In analogy with the classical terminology, we will refer to maps that do not have the typical splitting type as jumping curves.

Here we investigate the problem of compactifying the space of maps from $\mathbb{P}^{1}$ to $X$ of a specified splitting type for the vector bundle $E$. Our aim is to associate analogues of Gromov-Witten invariants to maps that have a given splitting type with respect to $E$. Intuitively, we would like these invariants to count the number of jumping rational curves of a fixed splitting type satisfying the appropriate number of constraints. If the partial flag varieties of the dual of $E$ over $X$ are homogeneous, the invariants we define will have this interpretation.

We remark that one can also make sense of higher genus jumping curves for a vector bundle $E$ on $X$. A smooth curve $C$ of genus $g$ on $X$ can be considered a jumping curve if the restriction of $E$ to $C$ has subbundles of unexpectedly high slope. Our approach allows us to define higher genus invariants of jumping curves. However, it is harder to give a satisfactory enumerative interpretation of these invariants in terms of the numbers of jumping curves.

A naive approach. The space $M_{0, m}(X, \beta)$ has a natural compactification given by the space of stable maps $\bar{M}_{0, m}(X, \beta)$. The existence of this compactification suggests a naive approach for defining the invariants of jumping curves.

Given $k$ integers $r_{1}, \ldots, r_{k}$, define $\bar{U}_{E, X, \beta}\left(r_{1}, \ldots, r_{k}\right)$ to be the closure of $U_{E, X, \beta}\left(r_{1}, \ldots, r_{k}\right)$, the subscheme of $M_{0, m}(X, \beta)$ parameterizing maps where $f^{*}(E)$ has splitting type $\left(r_{1}, \ldots, r_{k}\right)$ in $\bar{M}_{0, m}(X, \beta)$. Let $e v_{i}: \bar{M}_{0, m}(X, \beta) \rightarrow X$ denote the $i$-th evaluation morphism. Suppose that $\bar{U}_{E, X, \beta}\left(r_{1}, \ldots, r_{k}\right)$ has a virtual fundamental class supported in the expected dimension $D$. The expected dimension $D$ may be computed as follows. Set $s_{1}, \ldots, s_{k}$ to be the non-decreasing partition of $c_{1}(E) \cdot \beta$ subject to the condition that $\left|s_{i}-s_{j}\right| \leq 1$ for all $i, j$. The splitting type $s_{1}, \ldots, s_{k}$ corresponds to the most balanced possible splitting type, and it is the expected generic splitting type. The expected codimension of curves with any other splitting type is equal to the codimension of scrolls with that splitting type among all scrolls. Using Lemma 2.4 the expected dimension $D$ is given by

$$
-K_{X} \cdot \beta+\operatorname{dim}(X)+m-3+\sum_{j=0}^{k-1}(k-2 j-1)\left(s_{k-j}-r_{k-j}\right)-\sum_{i<j} \delta_{r_{i}}^{r_{j}}+\sum_{i<j} \delta_{s_{i}}^{s_{j}},
$$

provided that this expression is at least $m$. Otherwise, the expected dimension is -1 . 
Given the classes $\alpha_{1}, \ldots, \alpha_{m}$ of $m$ algebraic subvarieties of $X$ whose codimensions add up to $D$, we can define the $r_{1}, \ldots, r_{k}$ invariants of $E$ with respect to the classes $\alpha_{i}$ by the integral

$$
\int_{\left[\bar{U}_{E, X, \beta}\left(r_{1}, \ldots, r_{k}\right)\right]} \operatorname{virt} e v_{1}^{*} \alpha_{1} \cup \cdots \cup e v_{m}^{*} \alpha_{m} .
$$

There are two problems with this approach. First, it is far from obvious that these invariants are well-defined for arbitrary $X$ and $E$. Second, it is not clear in general how to compute these invariants. One can try to express the locus $\bar{U}_{E, X, \beta}\left(r_{1}, \ldots, r_{k}\right)$ as a degeneracy locus of a map of vector bundles on $\bar{M}_{0, m}(X, \beta)$. This calculation, except when $\bar{U}_{E, X, \beta}\left(r_{1}, \ldots, r_{k}\right)$ is a divisor 1 (see [Ran]), is difficult to perform because it is hard to describe the intersection of $\bar{U}_{E, X, \beta}\left(r_{1}, \ldots, r_{k}\right)$ with the boundary of $\bar{M}_{0, m}(X, \beta)$. Reducible curves usually contribute higherdimensional extraneous loci in the degeneracy locus calculations. Given these inherent difficulties in the naive approach, we will follow a different approach.

A classical enumerative geometry problem. Our method is inspired by a classical enumerative problem posed by I. Vainsencher: "How many cones over twisted cubics are there in $\mathbb{P}^{4}$ that intersect 16 general lines?" Levcovitz, Vainsencher and Xavier computed that there are 279,596,220 such cones using methods of classical algebraic geometry (see $[\mathrm{VX}]$ and $[\mathrm{LVX}]$ ). He raised the question whether there are other methods to verify this number. It would be especially desirable to answer such enumerative geometry problems without constructing an explicit compactification for every type of scroll one wants to count.

Since a cone over a twisted cubic is a one-parameter family of lines it induces a rational curve of Plücker degree three in the Grassmannian $G(2,5)$ of lines in $\mathbb{P}^{4}$. Unfortunately, not every rational cubic curve in the Grassmannian corresponds to a cone over a twisted cubic. The tautological bundle of the Grassmannian has non-balanced splitting when restricted to rational cubic curves arising from cones over twisted cubics (see C1]). We can reformulate Vainsencher's question as "How many cubic jumping curves of the tautological bundle intersect 16 general Schubert cycles $\sigma_{2}$ ?" where $\sigma_{2}$ is the cycle of lines meeting a line.

A cone over a twisted cubic induces a map not only to the Grassmannian $G(2,5)$, but also to the partial flag variety $F(1,2 ; 5)$. On every fiber of the cone there is a distinguished point, namely the cone point. We can thus compactify the space of cubic jumping curves as $\bar{M}_{0,0}(F(1,2 ; 5),(0,3))$ where the curve class $(0,3)$ denotes the Plücker degrees of the curves under the two projections $\pi_{i}: F(1,2 ; 5) \rightarrow G(i ; 5)$ for $i=1,2$. Note that a general cubic scroll also induces a curve in $F(1,2 ; 5)$. The point of intersection of the exceptional curve with the fiber gives a distinguished point on each fiber. The class of the resulting curve is $(1,3)$ as opposed to $(0,3)$. In the compactification given by $\bar{M}_{0,0}(F(1,2 ; 5),(0,3))$ the Gromov-Witten invariants are enumerative and they do provide an answer to the classical problem (see \$4.1).

The previous example can be extended to arbitrary Grassmannians $G(k, n)$. The jumping curves of the tautological bundle of $G(k, n)$ of a fixed splitting type have the expected dimension. In order to have the splitting type given by non-negative integers, it is more convenient to consider the jumping curves of the dual of the

\footnotetext{
${ }^{1}$ During the preparation of this note the author was informed that Cristina Martinez made some progress in computing the invariants for the Grassmannian $G(2,4)$ when the jumping curves form a divisor in the space of stable maps.
} 
tautological bundle. Given Schubert cycles whose codimensions sum to the dimension given by Lemma 2.4. we can define the naive Gromov-Witten invariant of jumping curves of type $\left(r_{1}, \ldots, r_{k}\right)$ for the dual of the tautological bundle to be the number of irreducible rational curves of type $\left(r_{1}, \ldots, r_{k}\right)$ that meet general representatives of the Schubert cycles. Note that the Plücker degree of such a curve is given by $\sum_{i=1}^{k} r_{i}$. The main observation is that these invariants are equal to ordinary Gromov-Witten invariants of certain partial flag varieties. This observation naturally leads to a definition of Gromov-Witten invariants of jumping curves for all smooth varieties $X$ and vector bundles $E$ on $X$.

More precisely, let $i_{1}, \ldots, i_{j}$ be a sequence of non-negative integers with $j \leq k$. Consider a splitting type given by the sequence of integers

$r_{1}=\cdots=r_{a_{1}}=i_{1}, \quad r_{a_{1}+1}=\cdots=r_{a_{1}+a_{2}}=i_{2}, \quad \ldots, \quad r_{k-a_{j}+1}=\cdots=r_{k}=i_{j}$.

We will use the shorthand $i_{1}^{a_{1}}, \ldots, i_{j}^{a_{j}}$ to denote this splitting sequence. Set $b_{i}=$ $\sum_{h=1}^{i} a_{h}$. Note that $b_{j}=k$. Let $F\left(b_{1}, \ldots, b_{j} ; n\right)$ denote the flag variety that parameterizes nested sequences of vector spaces $V_{1} \subset \cdots \subset V_{j} \subset V$ of dimensions $b_{i}$ of a fixed vector space $V$ of dimension $n$. This flag variety admits a projection

$$
\pi_{h}: F\left(b_{1}, \ldots, b_{j} ; n\right) \rightarrow G\left(b_{h} ; n\right)
$$

by sending $\left(V_{1}, \ldots, V_{j}\right)$ to $V_{h}$. The class of a curve in the flag variety is determined by the Plücker degrees of its $j$ projections. We will denote the curve classes by $\left(d_{1}, \ldots, d_{j}\right)$ where $d_{i}$ is the Plücker degree of the curve under the $i$-th projection.

Suppose $\gamma_{1}, \ldots, \gamma_{m}$ are classes of Schubert cycles in $G(k, n)$ whose codimensions sum to the dimension of jumping curves of type $i_{1}^{a_{1}}, \ldots, i_{j}^{a_{j}}$ of the dual of the tautological bundle given by Lemma 2.4. Let $\pi_{j}^{*} \gamma_{i}$ denote the pull-back of the Schubert cycles to the flag variety by the projection $\pi_{j}$. With this notation we can phrase the main observation as follows.

Proposition 1.1. Let $E$ be the dual of the tautological k-plane bundle on the Grassmannian $G(k, n)$. The naive Gromov-Witten invariant of splitting type $\left(i_{1}^{a_{1}}, \ldots, i_{j}^{a_{j}}\right)$ for $E$ associated to the Schubert classes $\gamma_{1}, \ldots, \gamma_{m}$ is equal to the Gromov-Witten invariant of $F\left(b_{1}, \ldots, b_{j} ; n\right)$ associated to the curve class $\left(a_{1} i_{1}, a_{1} i_{1}+a_{2} i_{2}, \ldots\right.$, $\left.\sum_{h} a_{h} i_{h}\right)$ and Schubert classes $\pi_{j}^{*} \gamma_{1}, \ldots, \pi_{j}^{*} \gamma_{m}$.

This proposition can be interpreted as a generalization of the "kernel-span technique" (see [BKT]). In fact Proposition 1 and its corollary in BKT immediately follow from Proposition 1.1 (see [C2] for an exposition from this point of view). We recall that the authors define the kernel of a curve as the intersection of all the $k$ planes parameterized by the curve. Unfortunately, the kernel of a rational curve $C$ in the Grassmannian $G(k, n)$ is almost always empty unless the curve has very small degree or is very special. We can replace the kernel by a more natural invariant: the sequence of minimal subscrolls associated to the curve. In case the minimal subscrolls are the vertices of a cone we recover the kernel. The advantage of using minimal subscrolls is that every irreducible rational curve in the Grassmannian has an associated sequence of minimal subscrolls.

We also remark that Proposition 1.1 explains the "unexpected" vanishing of a large collection of Gromov-Witten invariants of partial flag varieties. In order for a Gromov-Witten invariant not to vanish it is not enough for the codimensions of the classes $\gamma_{i}$ to add up to the dimension of the Kontsevich moduli space. The conditions must allow the existence of the appropriate collection of minimal subscrolls. 
Proposition 1.1 and the dimension counts of $₫ 2$ give refined vanishing results. For example, the $m$-pointed degree $d$ genus-zero invariants of $G(k, n)$, assuming that $m \geq 3,2 k \leq n$ and $d+k \leq n$, vanish unless

$$
d+\frac{m-3}{d} \leq(m-2) k .
$$

This was observed for $m=3$ in [BKT]. The subvariety parameterizing $(k+d)$ dimensional vector spaces that contain a $k$-dimensional subspace satisfying the Schubert condition $\lambda_{i}$ has codimension $\sum_{j} \max \left(\lambda_{i}^{j}-d, 0\right)$ in $G(k+d, n)$, where $\lambda_{i}^{j}$ denotes the parts of the partition defining the Schubert condition $\lambda_{i}$. The sum of the codimensions $\sum_{i, j} \max \left(\lambda_{i}^{j}-d, 0\right)$ cannot exceed the dimension of $G(k+d, n)$ since a scroll of dimension $k$ and degree $d$ can span at most a $(k+d-1)$ dimensional projective linear space. We thus obtain the more precise inequality $\sum_{i, j} \max \left(\lambda_{i}^{j}-d, 0\right) \leq(k+d)(n-k-d)$. Since the sum of the codimensions of $\lambda_{i}$, $\sum_{i, j} \lambda_{i}^{j}$, is equal to the dimension of the Kontsevich moduli space, the right hand side is bounded below by $k(n-k)+d n-m k d+m-3$. Rewriting this dimension estimate yields the claimed inequality.

Once we transform the question of computing the invariants of jumping curves to a question of computing ordinary Gromov-Witten invariants, many techniques become available for computing these invariants. At the end of the paper we include a list of invariants computed using the associativity relations in the quantum cohomology ring following DiFI. We remark that, in order to facilitate these computations, it would be useful to give an efficient Littlewood-Richardson rule for the big quantum-cohomology of partial flag varieties using the structure of minimal subscrolls (see C1]).

Proposition 1.1 provides a tool for computing the characteristic numbers of scrolls. Recall that the characteristic numbers are the numbers of scrolls that are incident to the appropriate number of general linear spaces. Since there is a one-to-one correspondence between rational scrolls $S_{r_{1}, \cdots, r_{k}}$ in $\mathbb{P}^{n}$ and irreducible rational curves in $G(k, n+1)$ on which the dual of the tautological bundle has splitting type $\left(r_{1}, \ldots, r_{k}\right)$ (except when the scroll is a balanced scroll of degree 1 or 2), Proposition 1.1 has the following corollary.

Corollary 1.2. Let $\left(r_{1}, \ldots, r_{k}\right)$ be equal to the splitting type $\left(i_{1}^{a_{1}}, \ldots, i_{j}^{a_{j}}\right)$. The characteristic numbers of scrolls $S_{r_{1}, \cdots, r_{k}}$ in $\mathbb{P}^{n}$ having $m$ fibers satisfying the Schubert conditions $\gamma_{1}, \ldots, \gamma_{m}$ are equal to the Gromov-Witten invariants of $F\left(b_{1}, \ldots\right.$, $\left.b_{j} ; n+1\right)$ for the curve class $\left(i_{1} a_{1}, \ldots, \sum_{h=1}^{j} i_{h} a_{h}\right)$ associated to the cohomology classes $\pi_{j}^{*} \gamma_{1}, \ldots, \pi_{j}^{*} \gamma_{m}$, except when the scroll is a balanced scroll of degree 1 or 2 .

If $X$ is a smooth projective variety and $E$ is a vector bundle of rank $k$, then, inspired by the case of the tautological bundle of the Grassmannian, we can define the invariants of jumping curves as the genus-zero Gromov-Witten invariants of $F_{X}\left(b_{1}, \ldots, b_{j} ; E^{*}\right)$, the variety of partial flags of the dual of the vector bundle $E$. These invariants, by virtue of being defined as Gromov-Witten invariants, obey all the axioms of Gromov-Witten theory. If $F_{X}\left(b_{1}, \ldots, b_{j} ; E^{*}\right)$ is homogeneous, then the invariants are enumerative. They count the number of jumping curves of a fixed splitting type.

One can also consider the higher genus invariants of $F_{X}\left(b_{1}, \ldots, b_{j} ; E^{*}\right)$. The Kontsevich moduli space of genus $g$ maps to $F_{X}\left(b_{1}, \ldots, b_{j} ; E^{*}\right)$ may be interpreted 
as a "compactification" of genus $g$ curves on which the restriction of $E$ has subbundles of given rank and degree. Unfortunately, the Kontsevich moduli spaces of higher genus maps are not well-behaved. The author does not know a way of extracting useful geometric information from these invariants. We will, therefore, restrict our attention to genus zero curves.

Although there is a vast literature pertaining to the jumping lines of bundles on $\mathbb{P}^{n}$, relatively little seems to have been written concerning higher degree jumping curves of a fixed splitting type or invariants of jumping curves for varieties other than $\mathbb{P}^{n}$. Z. Ran has given a formula for the invariants of jumping curves for bundles on $\mathbb{P}^{n}$ (satisfying suitable assumptions) provided that the jumping curves are of pure codimension one in the space of rational curves of degree $d[\mathrm{Ran}$. However, the author could not find a convenient reference for the invariants of higher degree jumping curves for even the Horrocks-Mumford bundle on $\mathbb{P}^{4}$. We include these invariants at the end of the paper.

Questions. There are many open problems concerning the existence of vector bundles on $\mathbb{P}^{n}$. Two of these outstanding problems are the Hartshorne and Grauert conjectures.

- Are there any indecomposible rank 2 vector bundles on $\mathbb{P}^{n}$ for $n \geq 5$ ? The nonexistence of such bundles is Hartshorne's conjecture and is related to the question of whether there are any codimension-two smooth subvarieties that are not complete intersections in $\mathbb{P}^{n}$ for $n \geq 6$ (see $[\mathrm{Hu}]$ ).

- Are there any unstable bundles of rank 2 on $\mathbb{P}^{n}$ for $n \geq 4$ other than direct sums of line bundles? The non-existence of such bundles is also conjectured.

It would be interesting to study the implications of the geometry of jumping curves to these questions. While jumping lines give some insight into the problems, they are too rigid to resolve them. Taking higher degree or even higher genus curves may give the added flexibility to obtain better results.

The organization of the paper. In $₫ 2$ we recall classical facts about the geometry of scrolls and flag varieties. In $₫ 3$ we define the invariants of jumping curves and prove their basic properties. In $\S 4$ we compute the invariants of jumping curves for the dual of the tautological bundle of the Grassmannians and the HorrocksMumford bundle.

\section{Preliminaries}

2.1. The geometry of rational normal scrolls. In this subsection we recall the basic facts about rational normal scrolls. The reader can consult [C1], [H] or [EH] for more details.

The scrolls $S_{r_{1}, \cdots, r_{k}}$. Let $r_{1} \leq \cdots \leq r_{k}$ be a sequence of non-negative integers not all equal to zero. We will reserve the letter $r$ for the sum $r=\sum_{i} r_{i}$. We denote the $k$-dimensional scroll of type $r_{1}, \ldots, r_{k}$ in $\mathbb{P}^{r+k-1}$ by $S_{r_{1}, \cdots, r_{k}}$. To construct $S_{r_{1}, \cdots, r_{k}}$ fix rational normal curves of degree $r_{i}$ in general linear spaces $\mathbb{P}^{r_{i}}$. Choose an isomorphism between each of the rational curves with an abstract $\mathbb{P}^{1}$. The scroll $S_{r_{1}, \cdots, r_{k}}$ is the union of the $(k-1)$-planes spanned by the points corresponding under the isomorphisms. We allow some $r_{i}$ to be zero. In that case we obtain cones over scrolls of smaller dimension. We say a scroll is balanced if $\left|r_{i}-r_{j}\right| \leq 1$ for all $i, j$. We say a scroll is perfectly balanced if $r_{i}=r_{j}$ for every $i$ and $j$. 
Abstractly a scroll is the projectivization of a vector bundle of rank $k$ on $\mathbb{P}^{1}$. Hence, we can express the variety as $X=\mathbb{P} E=\mathbb{P}\left(\mathcal{O}_{\mathbb{P}^{1}}\left(-r_{1}\right) \oplus \cdots \oplus \mathcal{O}_{\mathbb{P}^{1}}\left(-r_{k}\right)\right)$. If $\pi: X \rightarrow \mathbb{P}^{1}$ is the projection morphism, then the Chow ring of $X$ is generated by the pull-back of the point class $F$ from $\mathbb{P}^{1}$ and the class $H=\mathcal{O}_{\mathbb{P E}}(1)$ which restricts to the hyperplane class on every fiber of $\pi$. The following proposition elucidates the relation between scrolls and projectivization of vector bundles over $\mathbb{P}^{1}$ (see $[\mathrm{EH}]$ ).

Proposition 2.1. The scroll $S_{r_{1}, \cdots, r_{k}}$ is the image of $\mathbb{P}\left(\mathcal{O}_{\mathbb{P}^{1}}\left(-r_{1}\right) \oplus \cdots \oplus \mathcal{O}_{\mathbb{P}^{1}}\left(-r_{k}\right)\right)$ under the linear series $|H|$.

More generally, we will consider projections of $S_{r_{1}, \cdots, r_{k}}$ along centers disjoint from the scroll. Abusing notation we will denote these projections also by $S_{r_{1}, \cdots, r_{k}}$.

Definition 2.2. An effective class of the form $H+m F$ is called a section class.

A section class restricts to a hyperplane on a general fiber $F \cong \mathbb{P}^{k-1}$. In particular an irreducible curve in the class $(H+m F)^{k-1}$ is a section of the projective bundle $\pi: X \rightarrow \mathbb{P}^{1}$. The dimension of the space of global sections of a section class can be easily computed (see $[\mathrm{EH}]$ ).

Lemma 2.3. On $S_{r_{1}, \cdots, r_{k}}$ the dimension of the space of global sections of $H+m F$ is given by

$$
\sum_{i=1}^{k}\left(\max \left(-1, m+r_{i}\right)+1\right) .
$$

Minimal subscrolls of $S_{r_{1}, \cdots, r_{k}}$. A subscroll of $S_{r_{1}, \cdots, r_{k}}$ of dimension $s$ is a scroll $S_{b_{1}, \cdots, b_{s}} \subset S_{r_{1}, \cdots, r_{k}}$ dominating the base of $S_{r_{1}, \cdots, r_{k}}$. A subscroll of dimension $s$ has class $H^{k-s}+m H^{k-s-1} F$ for some integer $m$. The scroll $S_{r_{1}, \cdots, r_{k}}$ has minimal subscrolls of dimension $s$ for every $s<k$. By Lemma 2.3 $H-r_{k} F$ is the minimal effective section class since $H-\left(r_{k}+j\right) F$ is not effective for any $j>0$. The $(k-1)$-dimensional minimal subscroll is $S_{r_{1}, \ldots, r_{k-1}}$. If $r_{k}>r_{k-1}$, it is unique. We can inductively define the minimal subscroll of codimension $s$ as a minimal subscroll of a minimal subscroll of codimension $s-1$. If all the $r_{i}$ are distinct, the minimal subscrolls are unique. Although the minimal subscrolls have been defined inductively, a minimal subscroll of dimension $s$ is the smallest degree subscroll of dimension $s$ contained in $S_{r_{1}, \cdots, r_{k}}$.

The dimension of the space of subscrolls of $S_{r_{1}, \cdots, r_{k}}$ may be computed using the dimensions of the spaces of sections of $S_{r_{1}, \cdots, r_{k}}$. The dimension of the spaces of curves of degree $d$ in the class $H^{k-1}+(d-r) H^{k-2} F$ is given by

$$
\sum_{i=1}^{k}\left(\max \left(-1, d-r_{i}\right)+1\right)-1 .
$$

The dimension of the space of subscrolls of $S_{r_{1}, \cdots, r_{k}}$ of type $S_{t_{1}, \ldots, t_{s}}$ assuming that $t_{j} \geq r_{j}$ and $s<k$ is given by the following:

$$
\sum_{j=1}^{s} \sum_{i=1}^{k}\left(\max \left(-1, t_{j}-r_{i}\right)+1\right)-\sum_{j=1}^{s} \sum_{h=1}^{s}\left(\max \left(-1, t_{j}-t_{h}\right)+1\right) \text {. }
$$

In particular, if the splitting sequence is $\left(i_{1}^{a_{1}}, \ldots, i_{j}^{a_{j}}\right)$, then the minimal subscrolls of dimensions $b_{i}=\sum_{h=1}^{i} a_{h}$ are unique. However, when there are repetitions, the minimal subscrolls need not be unique. In fact, the dimension of the space of 
nested sequences of minimal subscrolls of every dimension $S^{1} \subset \cdots \subset S^{k}=S_{r_{1}, \cdots, r_{k}}$ on $S_{r_{1}, \cdots, r_{k}}$ is $\sum_{i<j} \delta_{r_{j}}^{r_{i}}$, where $\delta$ denotes the Kronecker delta function.

The dimension of the space of scrolls. The dimension $a$ of the automorphism group of $S_{r_{1}, \cdots, r_{k}}$ is :

$$
a=\sum_{j=0}^{k-1}(k-2 j-1) r_{k-j}+\left(\begin{array}{c}
k+1 \\
2
\end{array}\right)+2+\sum_{i<j} \delta_{r_{i}}^{r_{j}}
$$

(see Ch. 3 of $[\mathrm{H}]$ ). Knowing the dimension of the automorphism group allows us to determine the dimension of the locus in the Hilbert scheme parameterizing rational scrolls $S_{r_{1}, \cdots, r_{k}}$ in any projective space $\mathbb{P}^{N}$.

Lemma 2.4. The dimension of the locus of the Hilbert scheme parameterizing rational scrolls $S_{r_{1}, \cdots, r_{k}}$ in $\mathbb{P}^{N}$ is

$$
(N+1)(r+k)-1-a .
$$

Remark. Note that Lemma 2.4 also determines the dimension of the space of rational curves of splitting type $\left(r_{1}, \ldots, r_{k}\right)$ for the dual of the tautological bundle of the Grassmannian. By the universal property of Grassmannians, every scroll induces a rational curve in the Grassmannian. The type of the scroll determines the splitting type of the dual of the tautological bundle.

Finally observe that the dimension of the space of scrolls $S_{r_{1}, \cdots, r_{k}}$ with a choice of sequence of minimal subscrolls is given by

$$
(N+1)(r+k)-\sum_{j=0}^{k-1}(k-2 j-1) r_{k-j}-\left(\begin{array}{c}
k+1 \\
2
\end{array}\right)-3 .
$$

In particular, the dimension is maximized when the scrolls are as balanced as possible. As the splitting becomes more unbalanced the dimension strictly decreases.

2.2. Quantum cohomology of partial flag varieties. In this subsection we collect basic facts about the quantum cohomology of partial flag varieties. The reader can consult $\mathrm{Ci}$ ] and $\mathrm{FP}$ for more details.

Let $b_{1}<\cdots<b_{j}$ be a strictly increasing sequence of positive integers. Let $F\left(b_{1}, \ldots, b_{j} ; n\right)$ denote the partial flag variety parameterizing nested sequences of subspaces $V_{1}^{b_{1}} \subset \cdots V_{j}^{b_{j}} \subset V^{n}$ of dimension $b_{i}$ of a fixed vector space of dimension $n$. For convenience of notation we will set $b_{j+1}=n$ and $b_{0}=0$ in the formulae below. We remind the reader that the dimension of $F\left(b_{1}, \ldots, b_{j} ; n\right)$ is

$$
\sum_{i=1}^{j} b_{i}\left(b_{i+1}-b_{i}\right)
$$

Given a complete flag $F_{\bullet}=F_{0} \subset F_{1} \subset \cdots \subset F_{n}$ and a permutation $\omega$ on $n$ letters for which $\omega(i)<\omega(i+1)$ except possibly when $i=b_{h}$ for some $h$ between 1 and $j$, the Schubert variety $\Sigma_{\omega}$ is defined by

$$
\Sigma_{\omega}\left(F_{\bullet}\right)=\left\{V_{1} \subset \cdots \subset V_{j} \mid \operatorname{dim}\left(V_{i} \cap F_{h}\right) \geq \#\{\alpha \leq i: \omega(\alpha)>n-h\} \quad \forall i, h\right\} .
$$

The cohomology of $F\left(b_{1}, \ldots, b_{j} ; n\right)$ is generated by the Poincaré duals of the classes of Schubert cycles.

The flag variety $F\left(b_{1}, \ldots, b_{j} ; n\right)$ admits a projection morphism $\pi_{i}: F\left(b_{1}, \ldots, b_{j} ; n\right)$ $\rightarrow G\left(b_{i}, n\right)$ for every $i$ between 1 and $j$. The divisor class group of $F\left(b_{1}, \ldots, b_{j} ; n\right)$ 
is generated by the pull-backs $\pi_{i}^{*} \sigma_{1}$ of the generators of the divisor class group in $G\left(b_{i}, n\right)$ by these projections. Similarly the class $\beta$ of a curve $C$ in $F\left(b_{1}, \ldots, b_{j} ; n\right)$ is determined by the Plücker degrees $d_{1}, \ldots, d_{j}$ of its projections $\pi_{i}(C)$. We will, therefore, often give a curve class $\beta$ by specifying the degrees of the projections.

A variety $X$ is convex in the sense of Kontsevich if for every map $f: \mathbb{P}^{1} \rightarrow X$, the pull-back of the tangent bundle $T_{X}$ of $X$ to $\mathbb{P}^{1}$ is generated by global sections. Homogeneous spaces, in particular $F\left(b_{1}, \ldots, b_{j} ; n\right)$, are convex varieties.

Given a curve class $\beta \in H_{2}(X, \mathbb{Z}) /$ tors, we denote the Kontsevich moduli space of $m$-pointed, genus-zero stable maps to $X$ in the class $\beta$ by $\bar{M}_{0, m}(X, \beta)$. It is equipped with $m$ natural evaluation morphisms $e v_{1}, \ldots, e v_{m}: \bar{M}_{0, m}(X, \beta) \rightarrow X$. When $X$ is a convex variety, $\bar{M}_{0, m}(X, \beta)$ is the coarse moduli scheme associated to a smooth, proper Deligne-Mumford stack $\overline{\mathcal{M}}_{0, m}(X, \beta)$ of dimension

$$
-K_{X} \cdot \beta+\operatorname{dim} X+m-3 \text {. }
$$

The Kontsevich moduli space is smooth away from the locus of maps with automorphisms (see [FP]).

Specializing this discussion to the case of $F\left(b_{1}, \ldots, b_{j} ; n\right)$, if $\beta=\left(d_{1}, \ldots, d_{j}\right)$, then the dimension of $\bar{M}_{0, m}\left(F\left(b_{1}, \ldots, b_{j} ; n\right), \beta\right)$ is equal to

$$
\sum_{i=1}^{j} b_{i}\left(b_{i+1}-b_{i}\right)+\sum_{i=1}^{j} d_{i}\left(b_{i+1}-b_{i-1}\right)+m-3 .
$$

Given a collection of Schubert cycles $\sigma_{\omega_{1}}, \ldots, \sigma_{\omega_{m}}$ whose codimensions add up to the dimension of $\bar{M}_{0, m}\left(F\left(b_{1}, \ldots, b_{j} ; n\right), \beta\right)$, the Gromov-Witten invariant corresponding to these cycles is defined as

$$
I_{\left(F\left(b_{1}, \ldots, b_{j} ; n\right), \beta\right)}\left(\sigma_{\omega_{1}}, \ldots, \sigma_{\omega_{m}}\right)=\int_{\bar{M}_{0, m}\left(F\left(b_{1}, \ldots, b_{j} ; n\right), \beta\right)} e v_{1}^{*}\left(\sigma_{\omega_{1}}\right) \cup \cdots \cup e v_{m}^{*}\left(\sigma_{\omega_{m}}\right) .
$$

By Lemma 14 of $[\mathrm{FP}$ the Gromov-Witten invariants are equal to the number of maps from an irreducible $\mathbb{P}^{1}$ to $F\left(b_{1}, \ldots, b_{j} ; n\right)$ that intersect general representatives of the Schubert cycles $\sigma_{\omega_{i}}$.

More generally, we denote the variety of partial flag bundles of $E$ over $X$ of type $b_{1}, \ldots, b_{j}$ by $F_{X}\left(b_{1}, \ldots, b_{j} ; E\right)$. Recall that the latter variety parameterizes $V_{x}^{b_{1}} \subset \cdots V_{x}^{b_{j}}=E_{x}$ nested sequences of $b_{i}$-dimensional subspaces in the fibers $E_{x}$ of the vector bundle $E$. The dimension of $F_{X}\left(b_{1}, \ldots, b_{j} ; E\right)$ is given by

$$
\operatorname{dim} X+\sum_{i=1}^{j-1} b_{i}\left(b_{i+1}-b_{i}\right) .
$$

We denote the Grassmannian bundle of $b_{i}$-planes in the fibers of $E$ by $G_{X}\left(b_{i}, E\right)$. The flag variety $F_{X}\left(b_{1}, \ldots, b_{j} ; E\right)$ admits the projection $\pi_{X}: F_{X}\left(b_{1}, \ldots, b_{j} ; E\right) \rightarrow X$ and the projections

$$
\pi_{i}: F_{X}\left(b_{1}, \ldots, b_{j} ; E\right) \rightarrow G_{X}\left(b_{i}, E\right) .
$$

Let $\beta$ be a curve class in $H_{2}(X, \mathbb{Z})$. The class of a curve $C$ in $F_{X}\left(b_{1}, \ldots, b_{j} ; E\right)$ is determined by the classes of the projections $\pi_{i}(C)$ in $G_{X}\left(b_{i} ; E\right)$ and the class of the projection of the curve $\beta$ in $X$. We will denote the class of a curve in $F_{X}\left(b_{1}, \ldots, b_{j} ; E\right)$ by $\left(\beta, e_{1}, \ldots, e_{j}\right)$ where the $e_{i}$ are the degrees of the curve with respect to the first Chern class of the universal quotient bundle over $G_{X}\left(b_{i} ; E\right)$. Note that the last degree $e_{j}$ is given by $e_{j}=c_{1}(E) \cdot \beta$. 


\section{Gromov-Witten invariants of Jumping Curves}

In this section we define the Gromov-Witten invariants for jumping curves and prove that they are enumerative for the tautological bundle of Grassmannians.

Let $E$ be a rank $k$ vector bundle on a smooth projective variety $X$. Let $\left(i_{1}^{a_{1}}, \ldots\right.$, $i_{j}^{a_{j}}$ ) denote the splitting type

$$
r_{1}=\cdots=r_{a_{1}}=i_{1}, \quad \ldots, r_{k-a_{j}+1}=\cdots=r_{k}=i_{j}
$$

as in the introduction. Set $b_{h}=\sum_{i=1}^{h} a_{i}$ and $d_{h}=\sum_{s=1}^{h} i_{s} a_{s}$.

Given a splitting sequence $\left(i_{1}^{a_{1}}, \ldots, i_{j}^{a_{j}}\right)$ and a curve class $\beta \in H_{2}(X, \mathbb{Z})$, there is an associated curve class $\left(\beta, d_{1}, \ldots, d_{j}\right)$ in the partial flag bundle $F_{X}\left(b_{1}, \ldots, b_{j} ; E^{*}\right)$ of the dual bundle $E^{*}$ of the bundle $E$ over $X$. We will denote this curve class by $\beta_{i_{1}^{a_{1}}, \ldots, i_{j}{ }_{j}}$. The $m$-pointed genus-zero maps in the curve class $\beta_{i_{1}^{a_{1}}, \ldots, i_{j}{ }_{j}}$ have an expected dimension given by the formula

$$
D=c_{1}\left(T_{F_{X}\left(b_{1}, \ldots, b_{j} ; E^{*}\right)}\right) \cdot \beta_{i_{1}^{a_{1}}, \ldots, i_{j}^{a_{j}}}+\operatorname{dim} F_{X}\left(b_{1}, \ldots, b_{j} ; E^{*}\right)+m-3 .
$$

Let $\gamma_{1}, \ldots, \gamma_{m}$ be the classes of $m$ algebraic subvarieties of $X$ whose codimensions add up to $D$.

Definition 3.1 (Invariants of genus-zero jumping curves). We define the GromovWitten invariant associated to the curves in the class $\beta \in H_{2}(X, \mathbb{Z})$ of splitting type $i_{1}^{a_{1}}, \ldots, i_{j}^{a_{j}}$ for the vector bundle $E$ and classes $\gamma_{1}, \ldots, \gamma_{m}$ by the following formula:

$$
\begin{aligned}
& I_{\beta, i_{1}^{a_{1}}, \ldots, i_{j}^{a_{j}}}\left(\gamma_{1}, \ldots, \gamma_{m}\right)
\end{aligned}
$$

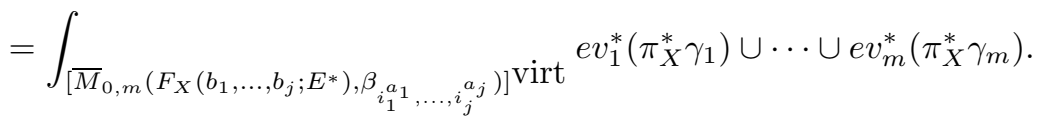

Remark. We note that $I_{\beta, i_{1}^{a_{1}}, \ldots, i_{j}^{a_{j}}}\left(\gamma_{1}, \ldots, \gamma_{m}\right)$ is well-defined because by assumption the sum of the codimensions of $\pi_{X}^{*} \gamma_{i}$ equals the expected dimension of $\bar{M}_{0, m}\left(F_{X}\left(b_{1}, \ldots, b_{j} ; E^{*}\right), \beta_{i_{1}^{a_{1}}, \ldots, i_{j}{ }_{j}}\right)$ and the corresponding Gromov-Witten invariant is well-defined.

We say that a map $f: \mathbb{P}^{1} \rightarrow F_{X}\left(b_{1}, \ldots, b_{j} ; E^{*}\right)$ in the class $\beta_{i_{1}^{a_{1}}, \ldots, i_{j}}$ is as balanced as possible if

$$
f^{*} \pi_{X}^{*} E \cong \bigoplus_{h=1}^{a_{1}} \mathcal{O}_{\mathbb{P}^{1}}\left(i_{1}\right) \oplus \bigoplus_{h=1}^{a_{2}} \mathcal{O}_{\mathbb{P}^{1}}\left(i_{2}\right) \oplus \cdots \oplus \bigoplus_{h=1}^{a_{j}} \mathcal{O}_{\mathbb{P}^{1}}\left(i_{j}\right)
$$

Let $M_{0, m}^{b a l}\left(F_{X}\left(b_{1}, \ldots, b_{j} ; E^{*}\right), \beta_{i_{1}^{a_{1}}, \ldots, i_{j}}{ }^{a_{j}}\right)$ denote the subscheme of the Kontsevich moduli space of stable maps $M_{0, m}\left(F_{X}\left(b_{1}, \ldots, b_{j} ; E^{*}\right), \beta_{i_{1}^{a_{1}}, \ldots, i_{j}{ }_{j}}\right)$ parameterizing maps that are as balanced as possible. This is an open (possibly empty) subscheme.

An easy computation shows that the expected dimension of

$$
\bar{M}_{0, m}\left(F_{X}\left(b_{1}, \ldots, b_{j} ; E^{*}\right), \beta_{i_{1}^{a_{1}}, \ldots, i_{j}} a_{j}\right)
$$

equals

$$
-K_{X} \cdot \beta+\operatorname{dim}(X)+m-3+\sum_{j=0}^{k-1}(k-2 j-1)\left(s_{k-j}-r_{k-j}\right)-\sum_{i<j} \delta_{r_{i}}^{r_{j}}+\sum_{i<j} \delta_{s_{i}}^{s_{j}}
$$


where $s_{1}, \ldots, s_{k}$ is the non-decreasing partition of $c_{1}(E) \cdot \beta$ subject to the condition that $\left|s_{i}-s_{j}\right| \leq 1$ for all $i, j$. Note that this is also the expected dimension of jumping curves of type $\left(i_{1}^{a_{1}}, \ldots, i_{j}^{a_{j}}\right)$ given in the introduction. Even for stable vector bundles on $\mathbb{P}^{n}$ and lines these expectations do not necessarily hold.

The theorem of Grauert and Mülich ensures that for a stable vector bundle on $\mathbb{P}^{n}$, the splitting type $\left(r_{1}, \ldots, r_{k}\right)$ on a general line satisfies $r_{i+1}-r_{i} \leq 1$ (see Chapter $2, \S 2$ of OSS ). However, there are stable vector bundles $E$ of rank 3 and $c_{1}(E)=0$ on $\mathbb{P}^{2}$ that have the splitting type $(-1,0,1)$ on a general line (see page 208 of OSS for an example).

There are analogues of the Grauert-Mülich Theorem determining the generic splitting types of stable vector bundles on $\mathbb{P}^{2}$ when restricted to conics (see Vit and Man]). However, in general, the actual dimensions of curves of a given splitting type seem to be unknown. Even when the splitting types for lines are as expected, the splitting types for other degree maps may have unexpectedly high dimension. For example, for the Horrocks-Mumford bundle, the expected dimension of degreetwo maps of splitting type $(-2,12)$ is -1 . However, the double covers of the lines of splitting type $(-1,6)$ give positive dimensional families of degree-two maps of splitting type $(-2,12)$. Hence, in general, the invariants we defined will be virtual. However, there are conditions that guarantee that for every splitting type the dimension of the jumping curves equals the expected dimension.

Proposition 3.2. $\quad$ (1) Between $M_{0, m}^{b a l}\left(F_{X}\left(b_{1}, \ldots, b_{j} ; E^{*}\right), \beta_{i_{1}^{a_{1}}, \ldots, i_{j}{ }_{j}}\right)$ and the locus of jumping curves for $E$ of type $\left(i_{1}^{a_{1}}, \ldots, i_{j}^{a_{j}}\right)$ in $M_{0, m}(X, \beta)$ there is a bijection.

(2) If the flag bundles $F_{X}\left(b_{1}, \ldots, b_{j} ; E^{*}\right)$ are homogeneous varieties, then for every jumping type the locus of rational curves having that jumping type for the vector bundle $E$ has the expected dimension. Moreover, the subscheme $M_{0, m}^{b a l}\left(F_{X}\left(b_{1}, \ldots, b_{j} ; E^{*}\right), \beta_{i_{1}^{a_{1}}, \ldots, i_{j}{ }_{j}}\right)$ forms an open dense subscheme of $\bar{M}_{0, m}\left(F_{X}\left(b_{1}, \ldots, b_{j} ; E^{*}\right), \beta_{i_{1}^{a_{1}}, \ldots, i_{j}{ }_{j}}\right)$; hence the latter gives a natural compactification of the space of m-pointed genus-zero jumping curves for $E$ of type $\left(i_{1}^{a_{1}}, \ldots, i_{j}^{a_{j}}\right)$.

Proof. Given a map $f: \mathbb{P}^{1} \rightarrow F_{X}\left(b_{1}, \ldots, b_{j} ; E^{*}\right)$ we obtain a map $f_{X}: \mathbb{P}^{1} \rightarrow X$ by taking the composition $\pi_{X} \circ f$ of $f$ with the projection $\pi_{X}$. Consequently, the morphism $\pi_{X}$ induces a morphism $\Pi_{X}: M_{0, m}\left(F_{X}\left(b_{1}, \ldots, b_{j} ; E^{*}\right), \beta_{i_{1}^{a_{1}}, \ldots, i_{j}{ }_{j}}\right) \rightarrow$ $M_{0, m}(X, \beta)$. We first argue that if $f$ is as balanced as possible, then $\pi_{X} \circ f$ is a jumping curve of type $\left(i_{1}^{a_{1}}, \ldots, i_{j}^{a_{j}}\right)$ for the bundle $E$.

The pull-back of $E$ by the composite map is given by $\left(\pi_{X} \circ f\right)^{*} E=f^{*} \pi_{X}^{*} E$. The relative flag variety has $j$ universal subbundles $U_{1} \subset \cdots \subset U_{j}$. We have that $U_{j} \cong \pi_{X}^{*} E^{*}$. Hence the $f^{*} U_{i}$ give subbundles of $f^{*} \pi_{X}^{*} E^{*}$ of rank $b_{i}$ and degree $-d_{i}$. Since the map $f$ is as balanced as possible, the pull-backs of the universal bundles decompose as

$$
f^{*} U_{s} \cong \bigoplus_{h=1}^{a_{1}} \mathcal{O}_{\mathbb{P}^{1}}\left(-i_{1}\right) \oplus \bigoplus_{h=1}^{a_{2}} \mathcal{O}_{\mathbb{P}^{1}}\left(-i_{2}\right) \oplus \cdots \oplus \bigoplus_{h=1}^{a_{s}} \mathcal{O}_{\mathbb{P}^{1}}\left(-i_{s}\right)
$$

By the assumption that the map is as balanced as possible this is true when $s=j$. Since the subbundles of rank $b_{s}$ and degree $-d_{s}$ in $f^{*} \pi_{X}^{*} E^{*}$ are unique, it follows for $1 \leq s<j$, as well. In particular, the splitting type of $\pi_{X} \circ f$ for $E$ is $\left(i_{1}^{a_{1}}, \ldots, i_{j}^{a_{j}}\right)$. 
Conversely, let $g: \mathbb{P}^{1} \rightarrow X$ be a morphism such that $g^{*} E=\bigoplus_{i=1}^{k} \mathcal{O}_{\mathbb{P}^{1}}\left(r_{i}\right)$. Assume that the integers $r_{i}$ are in increasing order. Let $b_{1}, \ldots, b_{j}=k$ be the collection of integers such that $r_{b_{i}}<r_{b_{i}+1}$, where the inequality is strict. Under these conditions the $g^{*} E^{*}$ have unique subbundles $F_{b_{i}}$ isomorphic to $\bigoplus_{i=1}^{b_{i}} \mathcal{O}_{\mathbb{P}^{1}}\left(-r_{i}\right)$. By the universal property of $F_{X}\left(b_{1}, \ldots, b_{j} ; E^{*}\right)$ there is a unique map $\tilde{g}: \mathbb{P}^{1} \rightarrow$ $F_{X}\left(b_{1}, \ldots, b_{j} ; E^{*}\right)$ such that $\pi_{X} \circ \tilde{g}=g$ and $\tilde{g}^{*} U_{i}=F_{b_{i}}$. Hence, $\tilde{g}$ is balanced. This gives the required bijection.

If $F_{X}\left(b_{1}, \ldots, b_{j} ; E^{*}\right)$ is convex in the sense of Kontsevich, then by Theorem 2 of FP the Kontsevich moduli spaces are of pure and expected dimension. In fact, they are smooth as Deligne-Mumford stacks. By $[\mathrm{KP}$ the Kontsevich moduli spaces of stable maps to homogeneous varieties are connected, hence irreducible. In the previous paragraph we showed that there is a bijection between stable maps in the class $\beta_{i_{1}^{a_{1}}, \ldots, i_{j}{ }_{j}}$ that are as balanced as possible and the jumping curves for $E$ of splitting type $\left(i_{1}^{a_{1}}, \ldots, i_{j}^{a_{j}}\right)$. This locus has dimension equal to the dimension of the Kontsevich space. It forms a dense open subset of it.

Proposition 3.3. Suppose the flag bundles $F_{X}\left(b_{1}, \ldots, b_{j} ; E^{*}\right)$ are homogeneous varieties. Then the Gromov-Witten invariant $I_{\beta, i_{1}^{a_{1}}, \ldots, i_{j}{ }_{j}}\left(\gamma_{1}, \ldots, \gamma_{m}\right)$ of jumping curves for $E$ is equal to the number of jumping curves of splitting type $\left(i_{1}^{a_{1}}, \ldots, i_{j}^{a_{j}}\right)$ for $E$ intersecting general varieties $\Gamma_{1}, \ldots, \Gamma_{m}$ whose classes are $\gamma_{1}, \ldots, \gamma_{m}$.

Proof. By Lemma 14 of $[\mathrm{FP}]$ the Gromov-Witten invariant

$$
I_{\left(F_{X}\left(b_{1}, \ldots, b_{j} ; E^{*}\right), \beta_{i_{1} a_{1}, \ldots, i_{j}} a_{j}\right)}\left(\pi_{X}^{*} \gamma_{1}, \ldots, \pi_{X}^{*} \gamma_{m}\right)
$$

is equal to the number of maps $f: \mathbb{P}^{1} \rightarrow F_{X}\left(b_{1}, \ldots, b_{j} ; E^{*}\right)$ such that $f\left(p_{i}\right) \in \pi_{X}^{-1} \Gamma_{i}$ where $\pi_{X}^{-1} \Gamma_{i}$ are general representatives of the Poincaré duals of the classes $\pi_{X}^{*} \gamma_{i}$.

By Proposition 3.2 there is a bijection between the jumping curves of type $\left(i_{1}^{a_{1}}, \ldots, i_{j}^{a_{j}}\right)$ and the maps in $M_{0, m}\left(F_{X}\left(b_{1}, \ldots, b_{j} ; E^{*}\right), \beta_{i_{1}^{a_{1}}, \ldots, i_{j}}\right)$ that are balanced. By the bijection given in the proof of Proposition 3.2 a curve that intersects general varieties $\Gamma_{1}, \ldots, \Gamma_{m}$ corresponds to a curve that intersects the varieties $\pi_{X}^{-1} \Gamma_{1}, \ldots, \pi_{X}^{-1} \Gamma_{m}$ and vice versa. If we assume that the non-balanced curves do not contribute to the Gromov-Witten invariants, then by this bijection the proposition follows.

The dimension counts in $\S 2.1$ prove that the non-balanced maps do not contribute to the Gromov-Witten invariants. The maps with irreducible domain in the class $\beta_{i_{1}^{a_{1}}, \ldots, i_{j}^{a_{j}}}$ that are not as balanced as possible give rise to maps with different splitting types in different flag varieties. Since their dimensions are strictly lower, by applying Lemma 14 of $[\mathrm{FP}$ we see that the corresponding Gromov-Witten invariants are zero. Hence, there cannot be maps with that splitting type that satisfy the constraints imposed by the classes $\gamma_{i}$. This concludes the proof of the proposition.

Proposition 1.1 is now a corollary of Proposition 3.3. When $X$ is the Grassmannian $G(k, n)$ and $E$ is the dual of the tautological $k$-plane bundle, then $F_{G(k, n)}\left(b_{1}\right.$, $\left.\ldots, b_{j} ; E^{*}\right)$ is simply the $j$-step partial flag variety $F\left(b_{1}, \ldots, b_{j} ; n\right)$. Since these varieties are homogeneous, Proposition 3.3 applies. We conclude that the GromovWitten invariant $I_{\beta, i_{1}^{a_{1}}, \ldots, i_{j}{ }_{j}}\left(\gamma_{1}, \ldots, \gamma_{m}\right)$ is equal to the number of jumping curves of class $\beta$ in $G(k, n)$ and splitting type $\left(i_{1}^{a_{1}}, \ldots, i_{j}^{a_{j}}\right)$ for the dual of the tautological 
bundle that intersect general Schubert cycles $\Gamma_{1}, \ldots, \Gamma_{m}$. This is the content of Proposition 1.1 in the Introduction.

We now establish the connection between the characteristic numbers of scrolls and the Gromov-Witten invariants of jumping curves for the dual of the tautological bundle of the Grassmannian. Let $\left(r_{1}, \ldots, r_{k}\right)$ be the splitting type given by $\left(i_{1}^{a_{1}}, \ldots, i_{j}^{a_{j}}\right)$.

Corollary 3.4. The number of scrolls $S_{r_{1}, \cdots, r_{k}}$ in $\mathbb{P}^{n-1}$ that have $m$ fibers that satisfy the Schubert conditions $\gamma_{1}, \ldots, \gamma_{m}$ is equal to the Gromov-Witten invariant $I_{F\left(b_{1}, \ldots, b_{j} ; n\right),\left(d_{1}, \ldots, d_{j}\right)}\left(\pi_{j}^{*} \gamma_{1}, \ldots, \pi_{j}^{*} \gamma_{m}\right)$ except when the scroll is a balanced scroll of degree 1 or 2 .

Proof. Let $f: \mathbb{P}^{1} \rightarrow G(k, n)$ be a map such that $f_{*}\left[\mathbb{P}^{1}\right]=d \sigma_{n-k, \ldots, n-k, n-k-1}$ where $\sigma_{n-k, \ldots, n-k, n-k-1}$ is the generator of $H_{2}(G(k, n), \mathbb{Z})$. Let $T$ denote the tautological bundle of $G(k, n)$. Suppose that the pull-back of the dual of the tautological bundle $f^{*} T^{*}$ has splitting type $\left(i_{1}^{a_{1}}, \ldots, i_{j}^{a_{j}}\right)$. The projectivization of the tautological bundle $\mathbb{P}\left(f^{*} T\right) \rightarrow \mathbb{P}^{1}$ naturally maps to $\mathbb{P}^{n-1}$. Consider the incidence correspondence

$$
\{(x,[\Lambda]): x \in \Lambda\} \subset \mathbb{P}^{n-1} \times G(k, n) .
$$

The second projection of the incidence correspondence gives the projectivization of the tautological bundle over $G(k, n)$. The first projection gives the desired map to $\mathbb{P}^{n-1}$. The image variety in $\mathbb{P}^{n-1}$ is either a scroll $S_{r_{1}, \cdots, r_{k}}$ (if the image spans an $(r+k-1)$-dimensional linear space) or a projection of a scroll $S_{r_{1}, \cdots, r_{k}}$.

Conversely, a scroll $S_{r_{1}, \cdots, r_{k}}$ in $\mathbb{P}^{n-1}$ induces a map $\mathbb{P}^{1} \rightarrow G(k, n)$ by the universal property of Grassmannians. The splitting type of the dual of the tautological bundle on the induced curve is $\left(r_{1}, \ldots, r_{k}\right)$. As long as the scroll is not balanced of degree 1 or 2 , each scroll induces a unique map to the Grassmannian. If the scroll is balanced of degree 2, then the scroll has two rulings by linear spaces, hence induces two distinct maps to the Grassmannian. Balanced scrolls of degree 1 admit many different scroll structures depending on their vertices. The corollary now follows from Proposition 1.1 and the fact that the two constructions just described are inverses of each other (except when the scrolls are balanced of degree 1 or 2 ).

Remark 1. The same technique can be used to compute the characteristic numbers of scrolls in other homogeneous varieties. For example, in order to compute the characteristic numbers of scrolls on a smooth quadric hypersurface one can repeat the discussion by replacing the Grassmannian $G(k, n)$ with the orthogonal Grassmannian.

Remark 2. Finally, observe that even if the flag bundles $F_{X}\left(b_{1}, \ldots, b_{j} ; E^{*}\right)$ are not homogeneous, as long as the Kontsevich moduli spaces

$$
\bar{M}_{0, m}\left(F_{X}\left(b_{1}, \ldots, b_{j} ; E^{*}\right), \beta_{i_{1}^{a_{1}}, \ldots, i_{j}^{a_{j}}}\right)
$$

are irreducible, reduced and of the expected dimension and $X$ is homogeneous, we can still obtain enumerative information from the invariants of jumping curves. In this case the invariants give the number of jumping curves (possibly counted with multiplicity). 


\section{Explicit COMPUTATIONS}

4.1. The invariants of jumping curves for the tautological bundle of $G(k, n)$. In this subsection we list the characteristic numbers of small degree unbalanced scrolls. By Corollary 3.4 these characteristic numbers are equal to certain Gromov-Witten invariants of partial flag varieties. The latter following [DiFI] can be computed using the associativity relations in the quantum cohomology ring. The numbers we list were computed using FARSTA $\underline{\mathrm{Kr}}$, a computer program that uses the associativity relations to determine the invariants from a few initial invariants.

The first table contains the number of surface scrolls $N\left(r_{1}, r_{2} ; p\right)$ in $\mathbb{P}^{3}$ of type $r_{1}, r_{2}$ that contain $p$ general points.

\begin{tabular}{|l|l|l|}
\hline$N(1,1 ; 9)=1$ & $N(2,2 ; 17)=1044120$ & $N(2,3 ; 21)=5335687360$ \\
\hline$N(0,2 ; 8)=4$ & $N(1,3 ; 16)=2059800$ & $N(1,4 ; 19)=6935529153$ \\
\hline$N(1,2 ; 13)=504$ & $N(0,4 ; 14)=310800$ & $N(0,5 ; 17)=181561120$ \\
\hline$N(0,3 ; 11)=900$ & & \\
\hline
\end{tabular}

The second table contains the number $N\left(r_{1}, r_{2} ; l, p\right)$ of surface scrolls in $\mathbb{P}^{4}$ of type $r_{1}, r_{2}$ that contain $p$ general points and meet $l$ general lines. We note that the quadric numbers may be computed by standard classical techniques. Some of these numbers were computed before in [C1] using degeneration techniques. The cubic number 279,596,220 has been recently computed by Levcovitz, Vainsencher and Xavier using techniques of classical algebraic geometry [LVX]. Other invariants of scrolls are available from the author upon request.

\begin{tabular}{|l|l|}
\hline$N(0,2 ; 12,0)=7440$ & $N(0,3 ; 16,0)=279596220$ \\
\hline$N(0,2 ; 10,1)=1180$ & $N(0,3 ; 14,1)=30177034$ \\
\hline$N(0,2 ; 8,2)=190$ & $N(0,3 ; 12,2)=3357774$ \\
\hline$N(0,2 ; 6,3)=30$ & $N(0,3 ; 10,3)=385008$ \\
\hline
\end{tabular}

4.2. The invariants of jumping curves for the Horrocks-Mumford bundle. In this final subsection we determine the invariants of jumping curves for the Horrocks-Mumford bundle. Let $\omega$ denote the hyperplane class in $\mathbb{P}^{4}$. Recall that the Horrocks-Mumford bundle is the unique (up to isomorphism and pull-back by projective linear transformations of $\mathbb{P}^{4}$ ) stable bundle on $\mathbb{P}^{4}$ with Chern polynomial $1+5 \omega+10 \omega^{2}[\mathrm{DS}$. The Horrocks-Mumford bundle occupies a special role in the theory of vector bundles of rank 2 on $\mathbb{P}^{n}$. We do not know any indecomposable vector bundles of rank 2 on $\mathbb{P}^{n}$ for $n>4$. On $\mathbb{P}^{4}$ the Horrocks-Mumford bundle provides an example of an indecomposable rank 2 vector bundle. One can obtain others by applying standard operations such as tensoring by a line bundle or pulling back the Horrocks-Mumford bundle via finite branched covers $f: \mathbb{P}^{4} \rightarrow \mathbb{P}^{4}$. However, all the indecomposable rank 2 bundles on $\mathbb{P}^{4}$ that we know essentially arise from the Horrocks-Mumford bundle via standard operations.

A theorem of Serre guarantees that the normal bundle of a smooth codimension 2 subvariety $X$ in $\mathbb{P}^{n}$ for $n \geq 3$ can be extended to a vector bundle on $\mathbb{P}^{n}$ if and only if the determinant of the normal bundle of $X$ is the restriction of $\mathcal{O}_{\mathbb{P}^{n}}(m)$ to $X$ for some $m$ (Theorem 5.1.1. of [OSS]). The Horrocks-Mumford bundle can be constructed as the extension of the normal bundle of an abelian surface of degree 


\begin{tabular}{|l|l|l|}
\hline$N(1,0,5 ; 0,0,2)=45$ & $N(1,0,5 ; 0,1,0)=30$ & $N(1,1,4 ; 0,0,4)=36$ \\
\hline$N(1,1,4 ; 0,1,2)=22$ & $N(1,1,4 ; 0,2,0)=14$ & $N(1,1,4 ; 1,0,1)=8$ \\
\hline$N(2,4,6 ; 0,0,10)=23250$ & $N(2,4,6 ; 0,1,8)=6065$ & $N(2,4,6 ; 0,2,6)=1645$ \\
\hline$N(2,4,6 ; 0,3,4)=456$ & $N(2,4,6 ; 0,4,2)=124$ & $N(2,4,6 ; 0,5,0)=35$ \\
\hline$N(2,4,6,1,0,7)=760$ & $N(2,4,6 ; 2,0,4)=37$ & $N(2,4,6 ; 3,0,1)=3$ \\
\hline$N(2,4,6,1,1,5)=230$ & $N(2,4,6 ; 1,3,1)=20$ & $N(2,4,6 ; 2,2,0)=3$ \\
\hline$N(2,4,6 ; 1,2,3)=71$ & $N(2,4,6 ; 2,1,2)=13$ & $N(2,3,7 ; 0,0,8)=47680$ \\
\hline$N(2,3,7 ; 0,1,6)=12340$ & $N(2,3,7 ; 0,2,4)=3308$ & $N(2,3,7 ; 0,3,2)=904$ \\
\hline$N(2,3,7 ; 0,4,0)=248$ & $N(2,3,7 ; 1,0,5)=1520$ & $N(2,3,7 ; 2,0,2)=68$ \\
\hline
\end{tabular}

10 embedded in $\mathbb{P}^{4}$ (see $[\mathrm{Hu}$ for additional constructions of the Horrocks-Mumford bundle).

Here we list some degree 1 and 2 invariants of the Horrocks-Mumford bundle. Longer lists of invariants are available from the author. We use the notation $N\left(d, r_{1}, r_{2} ; p, l, \lambda\right)$ to denote the Gromov-Witten invariant of degree $d$ rational curves with splitting type $\left(r_{1}, r_{2}\right)$ intersecting $p$ points, $l$ lines and $\lambda$ planes. We do not include the invariants for the generic splittings since the invariants of rational curves in $\mathbb{P}^{n}$ are well known (see for example $[\mathrm{V}]$ ).

These invariants were also computed using the associativity relations in the quantum cohomology ring of the projective bundle $\pi: \mathbb{P} E \rightarrow \mathbb{P}^{4}$ using FARSTA. As initial data we used the following invariants: There is a unique fiber of $\pi$ through a given point. There are not any fibers that intersect the inverse image of 2 lines or 4 planes since 2 lines or 4 planes do not have any common intersections in $\mathbb{P}^{4}$. There is a unique line with generic splitting containing 2 points. There are no conics with generic splitting intersecting $12-2 j$ planes and $j$ lines since the dimension of the space of conics in $\mathbb{P}^{4}$ is 11 . A sample of these invariants is given in the table above.

\section{ACKNOWLEDGEMENT}

It is a pleasure to thank T. Coates, J. de Jong, J. Harris, J. Starr and I. Vainsencher for enlightening conversations during the preparation of this note. Suggestions of I. Ciocan-Fontanine and the referee have greatly improved the style and the accuracy of this note. The author is grateful to A. Kresch for help with FARSTA.

\section{REFERENCES}

$[\mathrm{BHM}]$ W. Barth, K. Hulek, and R. Moore. Shioda's modular surface $S(5)$ and the HorrocksMumford bundle. In Vector bundles on algebraic varieties (Bombay, 1984), pages 35-106. Tata Inst. Fund. Res., Bombay, 1987.

[BKT] A. S. Buch, A. Kresch, and H. Tamvakis. Gromov-Witten invariants on Grassmannians. J. Amer. Math. Soc. 16(2003), 901-915. MR1992829 (2004h:14060)

[Ci] I. Ciocan-Fontanine. On quantum cohomology rings of partial flag varieties. Duke Math. J. 98(1999), 485-524. MR1695799 (2000d:14058)

[C1] I. Coskun. Degenerations of surface scrolls and the Gromov-Witten invariants of Grassmannians. J. Algebraic Geom. 15(2006), 223-284. MR2199064 (2006m:14073)

[C2] I. Coskun. A Littlewood-Richardson rule for two-step flag varieties. preprint.

[DS] W. Decker and F.-O. Schreyer. On the uniqueness of the Horrocks-Mumford bundle. Math. Ann. 273(1986), 415-443. MR824431 (87d:14009)

[DiFI] P. Di Francesco and C. Itzykson. Quantum intersection rings. In The moduli space of curves (Texel Island, 1994), volume 129 of Progr. Math., pages 81-148. Birkhäuser Boston, Boston, MA, 1995. MR1363054 (96k:14041a) 
[EH] D. Eisenbud and J. Harris. On varieties of minimal degree (a centennial account). In $A l$ gebraic geometry, Bowdoin, 1985 (Brunswick, Maine, 1985), volume 46 of Proc. Sympos. Pure Math., pages 3-13. Amer. Math. Soc., Providence, RI, 1987. MR927946 (89f:14042)

[FP] W. Fulton and R. Pandharipande. Notes on stable maps and quantum cohomology. In Algebraic geometry - Santa Cruz 1995, volume 62 Part 2 of Proc. Sympos. Pure Math., pages 45-96. Amer. Math. Soc., 1997. MR.1492534(98m:14025)

[GH] P. Griffiths and J. Harris. Principles of Algebraic Geometry. Wiley Interscience, 1978. MR507725 (80b:14001)

$[\mathrm{H}] \quad$ J. Harris. A bound on the geometric genus of projective varieties. Thesis, Harvard University (1978).

[HM] G. Horrocks and D. Mumford. A rank 2 vector bundle on $\mathbf{P}^{4}$ with 15, 000 symmetries. Topology 12(1973), 63-81. MR0382279 (52:3164)

$[\mathrm{Hu}] \mathrm{K}$. Hulek. The Horrocks-Mumford bundle. In Vector bundles in algebraic geometry (Durham, 1993), volume 208 of London Math. Soc. Lecture Note Ser., pages 139-177. Cambridge Univ. Press, Cambridge, 1995. MR1338416 (96g:14034)

[KP] B. Kim and R. Pandharipande. The connectedness of the moduli space of maps to homogeneous spaces. In Symplectic geometry and mirror symmetry (Seoul, 2000), pages 187-201. World Sci. Publishing, River Edge, NJ, 2001. MR1882330 (2002k:14021)

[Kr] A. Kresch. FARSTA, computer program. Available at http://www.maths.warwick.ac. $\mathrm{uk} / \sim \mathrm{kresch} / \mathrm{co} /$ farsta.html.

[LVX] D. Levcovitz, I. Vainsencher, and F. Xavier. Enumeration of cones over cubic scrolls. To appear in Israel J. Math.

[Man] M. Manaresi. On the jumping conics of a semistable rank two vector bundle on $\mathbf{P}^{2}$. Manuscripta Math. 69(1990), 133-151. MR1072985 (92b:14023)

[OSS] C. Okonek, M. Schneider, and H. Spindler. Vector bundles on complex projective spaces, volume 3 of Progress in Mathematics. Birkhäuser Boston, Mass., 1980. MR561910 (81b:14001)

[Ran] Z. Ran. The degree of the divisor of jumping rational curves. Q. J. Math. 52(2001), 367383. MR 1865907 (2002j:14009)

[VX] I. Vainsencher and F. Xavier. A compactification of the space of twisted cubics. Math. Scand. 91(2002), 221-243. MR1931571 (2003j:14073)

[V] R. Vakil. The enumerative geometry of rational and elliptic curves in projective space. $J$. Reine Angew. Math. 529(2000), 101-153. MR1799935(2001j:14072)

[Vit] A Vitter. Restricting semistable bundles on the projective plane to conics. Manuscripta Math. 114(2004), 361-383. MR2076453 (2005e:14066)

Mathematics Department, Massachusetts institute of Technology, Cambridge, MassachusetTs 02139

E-mail address: coskun@math.mit.edu 